\title{
Current Concepts in the Management of Infectious Endophthalmitis
}

\author{
Charles Q. Yu $\cdot$ Christopher N. Ta
}

Published online: 3 August 2013

(c) Springer Science + Business Media New York 2013

\begin{abstract}
Endophthalmitis can often result in blindness. The number of cases will most likely increase as intravitreal injections are performed more frequently. The prevention and treatment of endophthalmitis remains controversial. Recent data suggest that intracameral cefuroxime, which is now commercially available in Europe, reduces the risk of postoperative endophthalmitis. We here review the most up-to-date data on the incidence, prevention and treatment of infectious endophthalmitis.
\end{abstract}

Keywords Endophthalmitis - Intravitreal injection ·

Prophylaxis · Cataract surgery $\cdot$ Blebitis

\section{Introduction}

Endophthalmitis is intraocular inflammation generally caused by infection. Patients report blurred vision, redness, pain and eyelid swelling. On examination there is often hypopyon and vitritis. Timely diagnosis and treatment are key to preventing severe vision loss. Given the rarity of the condition, few randomized controlled clinical trials have been conducted. The prophylaxis of endophthalmitis remains controversial. We here conduct a Pubmed keyword search for "endophthalmitis" for articles published from April 2012 to 2013. We describe relevant clinical findings and provide our recommendation for the management of infectious endophthalmitis.

C. Q. Yu · C. N. Ta $(\bowtie)$

Stanford University School of Medicine, Stanford, CA, USA

e-mail: cta@stanford.edu

C. Q. Yu

e-mail: chyu83@gmail.com

\section{Injection Related Endophthalmitis}

Cases of infectious endophthalmitis following intravitreal injection will certainly increase with the sustained use of anti-VEGF injections in the treatment of numerous ocular conditions. In 2008 a total of 824,000 bevacizumab and ranizumab injections were given according to the Medicare part B database [1]. Taking into account non-Medicare usage and increasing use over the past five years, we estimate currently between 1 and 2 million intravitreal injections are given a year. Thus intravitreal injections are likely the second most common cause of endophthalmitis after cataract surgery. This is particularly concerning given the suggestion that endophthalmitis resulting from intravitreal injections is more severe than that following cataract surgery [2]. A recent study of rates in the UK found a per injection incidence of $0.025 \%$ [3]. A study from Boston demonstrated a rate of $0.029 \%$ [4]. This is comparable to the postoperative endophthalmitis rate following cataract surgery of $0.02-0.2 \%$ (Table 1 ). The repeated nature of anti-VEGF treatments increases the cumulative risk. For example, in a patient receiving a course of 24 injections, the likelihood of having at least one infection could range from $0.48 \%$ to a rather concerning $4.7 \%$.

Prophylaxis of intravitreal injection related endophthalmitis has been and remains controversial. Increase in the minimal inhibitory concentration of bacteria after routine antibiotic use before and after injection has been documented [5•]. A retrospective study examining fifteen thousand injections found a rate of endophthalmitis of $0.061 \%$ in patients receiving antibiotic for 5 days post injection, $0.084 \%$ in those receiving antibiotics immediately after injection and $0.034 \%$ in those not given antibiotics [6]. We agree with the authors who speculate that chronic use of antibiotics may select for more resistant species and strains 
Table 1 Rates of endophthalmitis

\begin{tabular}{|c|c|c|c|c|}
\hline Endophthalmitis type & Rate $(\%)$ & Prophylaxis & Study & Sample size \\
\hline \multirow[t]{2}{*}{ Intravitreal injection related } & 0.025 & Unknown & Lyall et al. [3] & $\sim 187,000$ \\
\hline & 0.029 & Unknown & Englander et al. [4] & 10,208 \\
\hline \multirow[t]{3}{*}{ Postcataract surgery } & 0.122 & Unknown & Keay et al. $[18 \bullet \bullet]$ & $3,280,966$ \\
\hline & 0.296 & Topical levofloxacin & ESCRS [19] & 8,103 \\
\hline & 0.062 & Intracameral cefuroxime & ESCRS [19] & 8,108 \\
\hline \multirow[t]{2}{*}{ Bleb related (per year) } & 0.17 & - & Alwitry et al. [28] & $\sim 9,961$ \\
\hline & 0.22 & - & Zahid et al. [30] & 285 \\
\hline Endogenous (with candidemia) & 1.6 & - & Oude Lashof et al. [35] & 370 \\
\hline
\end{tabular}

ESCRS European Society of Cataract and Refractive Surgeons

of bacteria. An update from the Diabetic Retinopathy Clinical Research Network found no significant difference in the rate of endophthalmitis with or without topical antibiotic [7]. Another retrospective study from the UK, however, found that antibiotics not given immediately prior to or after injection significantly increased the risk of endophthalmitis [3]. A retrospective study from Australia found a rate of $0.1 \%$, when the procedure was performed in the office, compared to $0 \%$ in an operating theater [8]. A study in Japan found zero cases of endophthalmitis in over 15,000 injections with the use of mask, povidone iodide and a course of pre-injection antibiotic [9]. A randomized trial comparing azithromycin to ofloxacin was recently completed in Spain, with the authors suggesting that azithromycin prophylaxis was significantly superior to ofloxacin prophylaxis in reducing rates of endophthalmitis ( 0.049 vs $0.12 \%$ ) [10]. This conclusion has been challenged because the data appears not to be significant with alternative statistical testing [11]. Another recent review covers each aspect of the injection technique without finding strong evidence for any particular method [12].

Although the use of topical antibiotic in the setting of intravitreal injection is controversial, we think it is important to prepare the eye with povidone iodide prior to each injection, wear a mask or avoid talking [13], and adhere to sterile technique. A preoperative course of antibiotics likely is not needed and could cause resistance given the typical dosing frequency of injections. Immediate preoperative antibiotic would not greatly reduce bacterial concentration in addition to povidone iodide, and immediate post operative topical antibiotic would not adequately penetrate the vitreous [14]. Conducting all injections in an operating theater is impractical. There are numerous variations among ophthalmologists with regard to speculum type and usage, conjunctival displacement, and location/quadrant of injection. We do not feel there is adequate evidence to support or argue against any particular technique.

When a case of post injection endophthalmitis is suspected, the patient should undergo vitreous sampling and antibiotic injection. It is unclear whether the results of the Endophthalmitis Vitrectomy Study (EVS) can guide treatment for injection related endophthalmitis [15]. Clinicians in New York retrospectively examined cases of endophthalmitis that were treated with tap and inject with or without vitrectomy. Clinical outcomes appeared worse for those that underwent vitrectomy, but the authors observed these had more severe cases of endophthalmitis and made no recommendations regarding the utility of vitrectomy [16]. There is insufficient evidence at this time to make conclusions upon the role of vitrectomy. There have been reports of post injection inflammatory endophthalmitis, however, the authors suggest that there is no reliable way to distinguish between post injection inflammation and infection [17]. In general, post injection inflammation should be treated aggressively with vitreous sampling and antibiotic injection.

\section{Post Cataract Endophthalmitis}

Three million cataract surgeries are performed each year in the United States, and; therefore, it is the most common procedure associated with endophthalmitis. A large metaanalysis found that the rate of post cataract endophthalmitis to be $0.265 \%$ in the early part of the last decade. Recent analysis of 2003-2004 Medicare data estimated a rate of $0.122 \%$ after examining over 3.2 million surgeries [18••]. These numbers trend higher than estimated rates of post injection endophthalmitis, reflecting the longer and more complex nature of cataract surgery compared to intravitreal injection. Although intravitreal injections have a lower per injection rate of endophthalmitis than cataract surgery, the repeated injections increase the overall risk of endophthalmitis for each patient.

It has long been widely accepted that topical povidone iodide is an effective prophylaxis for cataract surgeries. In addition, the results of the 2007 European Society of Cataract and Refractive Surgeons (ESCRS) study, a 
randomized clinical trial enrolling over 16,000 patients, demonstrated that intracameral cefuroxime is also an effective prophylaxis. This study showed a reduction in the incidence of post cataract endophthalmitis from 0.296 to $0.062 \%$ [19] (Table 1). The popularity of intracameral cefuroxime in Europe was boosted by this study. A retrospective study from France described a rather high rate of $1.238 \%$ without cefuroxime versus $0.044 \%$ with intracameral cefuroxime [20]. Similar work from Singapore demonstrated a rate of $0.01 \%$ with intracameral cefazolin as compared to $0.064 \%$ in the control group [21]. These and other studies point strongly to the efficacy of intracameral antibiotic prophylaxis. This may reflect the ability of the intracameral delivery method to deliver a more effective bacterial killing dose than topical treatment.

Adoption of intracameral prophylaxis in the United States has been slow given concerns for formulation, contamination, toxicity and the lack of commercially available cefuroxime preparation designed for intraocular use. A recent report detailed a series of patients injected with cefuroxime at an incorrectly formulated dose of $50 \mathrm{mg} / \mathrm{ml}$ and documented numerous complications including corneal edema, retinal pigmentary changes, ERG signal attenuation and severe vision loss [22]. However, cefuroxime designed for intracameral use, Aprokam ${ }^{\circledR}$ has been approved in the European Union [23•]. This will allay formulation fears and further increase the popularity of cefuroxime in the EU. In the mean time some large health systems such as Kaiser Permanente in California have begun to routinely use intracameral antibiotic prophylaxis with good results [24•]. Their study demonstrated an endophthalmitis rate of $0.313 \%$ with postoperative antibiotic drops, $0.143 \%$ with intracameral cefuroxime for those without cephalosporin allergy or capsular rupture, and $0.014 \%$ with the combined use of intracameral cefuroxime routinely along with intracameral moxifloxacin or vancomycin as back up for patients with allergies or capsular rupture.

The treatment of post cataract endophthalmitis has long been guided by the Early Vitrectomy Study (EVS) [15]. This randomized prospective study found that early vitrectomy reduced severe vision loss in patients with light perception or worse vision as compared to vitreous sampling and antibiotic injection. There was no benefit from early vitrectomy in patients with hand motion or better vision. This has become the standard of care. We emphasize that clinicians should follow the published procedure for checking vision, with hand motion being tested at a distance of $60 \mathrm{~cm}$, with a lamp directed from behind the patient towards the examiner's hand. The patient should be able to identify whether the examiner's hand is moving up or down, left or right, or not moving four out of five times to be considered to have hand motion vision. The EVS recommended injection of vancomycin and amikacin antibiotics. However, due to the potential retinal toxicity of amikacin, ophthalmologists have shifted to using ceftazidime instead of amikacin, and recent work confirms that these remain appropriate medication choices [25].

Controversy exists as to whether treatment with steroid is helpful in treatment of post cataract endophthalmitis. A recent trial randomized 32 patients with post cataract, bleb related and "other" causes of endophthalmitis to intravitreal ceftazidime, vancomycin and placebo and 30 patients to ceftazidime, vancomycin and dexamethasone [26]. They found no significant difference in visual outcomes at two weeks and two to four months. There was, however, a trend towards more visual improvement in steroid treated post cataract subgroup.

Delayed post operative endophthalmitis is defined as endophthalmitis occurring more than six weeks after surgery. It generally presents with better vision and less severe signs than acute postoperative endophthalmitis. A retrospective case series found this entity to be caused by Propionibacterium acnes in $42 \%$, fungal in $27 \%$, gram negative bacteria in $12 \%$, gram positive bacteria in $12 \%$, and atypical mycobacterium in $8 \%$ of eyes [27]. The authors recommended careful culture of suspected cases including anaerobic and fungal culture. This study found that, in cases of chronic endophthalmitis that recurred after injection of antibiotics, nearly all benefited from vitrectomy with removal of the capsule and IOL removal or exchange.

\section{Bleb Associated Endophthalmitis}

The presence of a filtering bleb allows for relative ease of access to the intraocular media and, thus, is a risk factor for endophthalmitis. Several retrospective studies examining this topic have recently been published. A study of the British Ophthalmic Surveillance Unit data examined 17 cases and calculated an annual rate of bleb associated endophthalmitis of $0.17 \%$ [28]. Half of patients in this study had outcomes of light perception or worse vision. A study from China confirmed findings of poor outcomes from bleb associated endophthalmitis, with only $38.7 \%$ of their 31 patients achieving better than 20/400 vision [29]. Analysis of long term complications in 285 patients randomized to trabeculectomy from the Collaborative Initial Glaucoma Treatment Study demonstrated a five year risk of endophthalmitis of $1.1 \%$ [30]. The annual risk of bleb associated endophthalmitis appears similar to that of one cataract surgery.

It is generally agreed upon that the results of the EVS are not applicable to cases of bleb associated endophthalmitis. It is suggested that given the generally poor outcomes, treatment for bleb related endophthalmitis should be more aggressive than that for post cataract endophthalmitis. The aforementioned study from China suggested that vitrectomy was of benefit to their patients with vision at the level of hand 
motion or worse. However, given the lack of prospective studies, there is no consensus regarding the level of vision at which a vitrectomy would be indicated. It is also clear that bleb leakage is a risk factor for infection and that any leaks which do not resolve in the short term should be surgically corrected.

\section{Endogenous Endophthalmitis}

Endogenous sources of infectious organisms can reach the intraocular media through the bloodstream and is generally associated with serious medical illness and immunosuppression [31]. In the United States fungi and molds are typically responsible. A case series examining 65 eyes with fungal endophthalmitis found visual acuity of better than 20/200 was achieved with treatment in only $50.8 \%$ [32]. Most of the patients underwent vitrectomy, and a high percentage (29) had retinal detachment. This is the first report of the rate of retinal detachment after vitrectomy in endogenous fungal endophthalmitis. No cases of endophthalmitis in this series were identified based on routine ocular screening after septicemia. A series examining 44 cases in the UK found vision better than 20/200 in $67 \%$ of cases. Early vitrectomy was found to be helpful in reducing the rate of retinal detachment, though not significantly improving final visual outcomes [33]. A case series of 90 eyes from Korea showed that $48.4 \%$ of endogenous endophthalmitis was caused by Klebsiella pneumonia as is typical of Asian countries. They also suggested that early vitrectomy may be helpful [34].

Ocular screening is recommended in the infectious disease literature for all patients with positive blood cultures for Candida regardless of symptoms. A study from 2011 showed that in 370 patients with positive Candida blood culture, ocular involvement occurred in $16 \%$ and endophthalmitis in $1.6 \%$ [35]. However, it is suggested that in adults without impaired consciousness who are asymptomatic, routine screening is of very low yield and perhaps unnecessary after a systemic course of treatment. A Swedish study of 144 patients with candidemia who survived more than 60 days found no cases of late onset ocular candidiasis in patients who had received at least 14 days of systemic antifungal treatment [36]. Prospective studies are necessary to delineate specific indications for routine ocular screening in patients with septicemia. The treatment of endogenous fungal endophthalmitis is generally systemic antifungal therapy given the underlying mechanism. Both voriconazole and amphotericin B appear effective [35, 37]. Intraocular injections may be of benefit when vitritis is noted. It is unclear when vitrectomy is helpful.

\section{Molecular Diagnosis}

Identification of the organism causing endophthalmitis can be very helpful in guiding treatment. However, given the rapid clinical course, cultures often take too long to be immediately helpful. For injection related endophthalmitis, cultures were positive in $59.6 \%$ of cases [3]. Fungal endophthalmitis was culture positive in $44 \%$ [32]. Postcataract endophthalmitis was culture positive in $42 \%$ [24•]. These numbers can vary from study to study, but clearly a significant proportion of cases are culture negative. There has been much work in recent years to develop reliable molecular methods to hasten the identification of causative organisms. A group in Japan has developed real time PCR for diagnosis of fungal endophthalmitis. They reported development of fungal endophthalmitis in 10/11 patients who were positive for fungal DNA with real time quantitative PCR techniques (RT PCR) [38]. Another group reported use of RT PCR in 64 cases of post cataract endophthalmitis. PCR yielded positive results in $66 \%$ of patients, as compared to $34 \%$ with traditional cultures. One patient who was culture positive for Nocardia was not identified by PCR. All cases positive for both PCR and culture identified the same organism [39]. With time molecular techniques will become a useful adjunct in the management of endophthalmitis by allowing for early identification of organisms.

\section{Conclusion}

Endophthalmitis is a devastating disease but there is hope for improved prevention, management and outcomes. Antibacterial prophylaxis is proven and effective. New techniques for microbiological identification are being developed. Improved treatment of endophthalmitis can potentially result in fewer patients with vision loss due to endophthalmitis.

Disclosure Charles Q. Yu declares that he has no conflict of interest. Christopher N. Ta is a consultant to Bausch and Lomb.

Human and Animal Rights and Informed Consent This article does not contain any studies with human or animal subjects performed by any of the authors.

\section{References}

Papers of particular interest, published recently, have been highlighted as:

- Of importance

-. Of major importance 
1. Brechner RJ, Rosenfeld PJ, Babish JD, Caplan S. Pharmacotherapy for neovascular age-related macular degeneration: an analysis of the $100 \% 2008$ Medicare fee-for-service part B claims file. Am J Ophthalmol. 2011;151(5):887-95 e1.

2. Simunovic MP, Rush RB, Hunyor AP, Chang AA. Endophthalmitis following intravitreal injection versus endophthalmitis following cataract surgery: clinical features, causative organisms and post-treatment outcomes. $\mathrm{Br} \mathrm{J}$ Ophthalmol. 2012;96(6): 862-6.

3. Lyall DA, Tey A, Foot B, et al. Post-intravitreal anti-VEGF endophthalmitis in the United Kingdom: incidence, features, risk factors, and outcomes. Eye (Lond). 2012;26(12):1517-26.

4. Englander M, Chen TC, Paschalis EI, et al. Intravitreal injections at the Massachusetts Eye and Ear Infirmary: analysis of treatment indications and postinjection endophthalmitis rates. $\mathrm{Br} \mathrm{J}$ Ophthalmol. 2013;97(4):460-5.

5. - Yin VT, Weisbrod DJ, Eng KT, et al. Antibiotic resistance of ocular surface flora with repeated use of a topical antibiotic after intravitreal injection. JAMA Ophthalmol 2013;131(4):456-61. This prospective clinical trial demonstrated increased MIC after use of topical antibiotic prophylaxis for intravitreal injections, suggesting that such prophylaxis be avoided.

6. Cheung CS, Wong AW, Lui A, et al. Incidence of endophthalmitis and use of antibiotic prophylaxis after intravitreal injections. Ophthalmology. 2012;119(8):1609-14.

7. Bhavsar AR, Stockdale CR, Ferris FL III, et al. Update on risk of endophthalmitis after intravitreal drug injections and potential impact of elimination of topical antibiotics. Arch Ophthalmol. 2012;130(6):809-10.

8. Abell RG, Kerr NM, Allen P, Vote BJ. Intravitreal injections: is there benefit for a theatre setting? Br J Ophthalmol. 2012;96(12): 1474-8.

9. Shimada H, Hattori T, Mori R, et al. Minimizing the endophthalmitis rate following intravitreal injections using $0.25 \%$ povidone-iodine irrigation and surgical mask. Graefes Arch Clin Exp Ophthalmol 2013

10. Romero-Aroca P, Sararols L, Arias L, et al. Topical azithromycin or ofloxacin for endophthalmitis prophylaxis after intravitreal injection. Clin Ophthalmol. 2012;6:1595-9.

11. Stewart MW, Stewart ML. Topical azithromycin or ofloxacin for endophthalmitis. Clin Ophthalmol. 2013;7:35-6.

12. Fagan XJ, Al-Qureshi S. Intravitreal injections: a review of the evidence for best practice. Clin Experiment Ophthalmol. 2012;41(5):500-7.

13. Schimel AM, Scott IU, Flynn HW Jr. Endophthalmitis after intravitreal injections: should the use of face masks be the standard of care? Arch Ophthalmol. 2011;129(12):1607-9.

14. Moss JM, Sanislo SR, Ta CN. A prospective randomized evaluation of topical gatifloxacin on conjunctival flora in patients undergoing intravitreal injections. Ophthalmology. 2009;116(8): 1498-501.

15. Results of the Endophthalmitis Vitrectomy Study. A randomized trial of immediate vitrectomy and of intravenous antibiotics for the treatment of postoperative bacterial endophthalmitis. Endophthalmitis Vitrectomy Study Group. Arch Ophthalmol. 1995;113(12): 1479-96.

16. Chaudhary KM, Romero JM, Ezon I, et al. Pars plana vitrectomy in the management of patients diagnosed with endophthalmitis following intravitreal anti-vascular endothelial growth factor injection. Retina. 2013;33(7):1407-16.

17. Shah CP, Garg SJ, Vander JF, et al. Outcomes and risk factors associated with endophthalmitis after intravitreal injection of anti-vascular endothelial growth factor agents. Ophthalmology. 2011;118(10):2028-34.

18. • Keay L, Gower EW, Cassard SD, et al. Postcataract surgery endophthalmitis in the United States: analysis of the complete
2003-2004 Medicare database of cataract surgeries. Ophthalmology 2012;119(5):914-22. This analysis of Medicare claims data examined 3,280,966 cataract surgeries to calculate a rate of endophthalmitis of $0.122 \%$ per cataract surgery. Being male and of older age were among risk factors.

19. Prophylaxis of postoperative endophthalmitis following cataract surgery. Results of the ESCRS multicenter study and identification of risk factors. J Cataract Refract Surg. 2007;33(6):978-88.

20. Barreau G, Mounier M, Marin B, et al. Intracameral cefuroxime injection at the end of cataract surgery to reduce the incidence of endophthalmitis: French study. J Cataract Refract Surg. 2012; 38(8):1370-5.

21. Tan CS, Wong HK, Yang FP. Epidemiology of postoperative endophthalmitis in an Asian population: 11-year incidence and effect of intracameral antibiotic agents. J Cataract Refract Surg. 2012;38(3):425-30.

22. Olavi P. Ocular toxicity in cataract surgery because of inaccurate preparation and erroneous use of $50 \mathrm{mg} / \mathrm{ml}$ intracameral cefuroxime. Acta Ophthalmol. 2012;90(2):e153-4.

23. - Keating GM. Intracameral cefuroxime: prophylaxis of postoperative endophthalmitis after cataract surgery. Drugs 2013;73(2): 179-86. This article describes the commercial availability of cefuroxime formulated for intracameral use in Europe.

24. - Shorstein NH, Winthrop KL, Herrinton LJ. Decreased postoperative endophthalmitis rate after institution of intracameral antibiotics in a Northern California eye department. J Cataract Refract Surg 2013;39(1):8-14. This study describes promising retrospective results in a large healthcare system using intracameral antibiotic prophylaxis for cataract surgery in the United States.

25. Schimel AM, Miller D, Flynn HW Jr. Endophthalmitis isolates and antibiotic susceptibilities: a 10-year review of culture-proven cases. Am J Ophthalmol. 2013;156(1):50-2.

26. Albrecht E, Richards JC, Pollock T, et al. Adjunctive use of intravitreal dexamethasone in presumed bacterial endophthalmitis: a randomised trial. Br J Ophthalmol. 2011;95(10):1385-8.

27. Shirodkar AR, Pathengay A, Flynn HW Jr, et al. Delayed- versus acute-onset endophthalmitis after cataract surgery. Am J Ophthalmol. 2012;153(3):391-8 e2.

28. Alwitry A, King AJ. Surveillance of late-onset bleb leak, blebitis and bleb-related endophthalmitis - a UK incidence study. Graefes Arch Clin Exp Ophthalmol. 2012;250(8):1231-6.

29. Ye H, Sun X, Gan D, et al. Bleb-associated endophthalmitis in a Chinese population (2003-2010): clinical characteristics and visual outcome. Eur J Ophthalmol. 2012;22(5):719-25.

30. Zahid S, Musch DC, Niziol LM, Lichter PR. Risk of endophthalmitis and other long-term complications of trabeculectomy in the Collaborative Initial Glaucoma Treatment Study (CIGTS). Am J Ophthalmol. 2013;155(4):674-80 e1.

31. Jackson TL, Eykyn SJ, Graham EM, Stanford MR. Endogenous bacterial endophthalmitis: a 17-year prospective series and review of 267 reported cases. Surv Ophthalmol. 2003;48(4):403-23.

32. Lingappan A, Wykoff CC, Albini TA, et al. Endogenous fungal endophthalmitis: causative organisms, management strategies, and visual acuity outcomes. Am J Ophthalmol. 2012;153(1):162-6 e1.

33. Sallam A, Taylor SR, Khan A, et al. Factors determining visual outcome in endogenous Candida endophthalmitis. Retina. 2012; 32(6):1129-34.

34. Lee S, Um T, Joe SG, et al. Changes in the clinical features and prognostic factors of endogenous endophthalmitis: 15 years of clinical experience in Korea. Retina. 2012;32(5):977-84.

35. Oude Lashof AM, Rothova A, Sobel JD, et al. Ocular manifestations of candidemia. Clin Infect Dis. 2011;53(3):262-8.

36. Blennow O, Tallstedt L, Hedquist B, Gardlund B. Duration of treatment for candidemia and risk for late-onset ocular candidiasis. Infection. 2013;41(1):129-34. 
37. Troke P, Obenga G, Gaujoux T, et al. The efficacy of voriconazole in 24 ocular Fusarium infections. Infection. 2013;41(1): $15-20$.

38. Ogawa M, Sugita S, Watanabe K, et al. Novel diagnosis of fungal endophthalmitis by broad-range real-time PCR detection of fungal 28S ribosomal DNA. Graefes Arch Clin Exp Ophthalmol. 2012;250(12):1877-83.

39. Joseph CR, Lalitha P, Sivaraman KR, et al. Real-time polymerase chain reaction in the diagnosis of acute postoperative endophthalmitis. Am J Ophthalmol. 2012;153(6):1031-7 e2. 